\title{
Multilayer approach to minimax theorems
}

\section{Yen-Cherng Lin ${ }^{1 *}$ and Hang-Chin Lai ${ }^{2}$}

${ }^{\text {*Correspondence: }}$ yclin@mail.cmu.edu.tw

${ }^{1}$ Department of Occupational Safety and Health, College of Public Health, China Medical University, Taichung, 40402, Taiwan

Full list of author information is available at the end of the article

\begin{abstract}
In this research, a multihierarchical methodology works by taking a whole intersection property to establish a fixed point theorem. Using such a whole intersection property to let multihierarchical procedures of set-valued mappings can work on a Hausdorff topological vector space. Some examples are proposed in order to illustrate our theory.
\end{abstract}

MSC: $90 \mathrm{C} 47 ; 58 C 06$

Keywords: minimax theorems; fixed points; whole intersection

\section{Introduction and preliminaries}

In 1961, on the basis of the KKM theorem, Fan established the celebrated minimax theorem $[1,2]$ by employing a whole intersection theorem. Since then, fruitful extensions for Fan's minimax theorem were established [3-7]. It is important in mathematical economics and game theory; in the meantime, it has been very useful in many applications in convex and nonlinear analysis. One of important extensional directions is the multihierarchical approach. A large number of papers [3, 4, 8-12] have developed along this direction and applied the concept of multihierarchical approach to either the whole intersection property or to minimax theorems for real-valued functions. In the meanwhile, $\mathrm{Ha}$ [3] recently established a minimax theorem ([3], Theorem 3.1), which uses the following whole intersection theorem.

Theorem A Let $X, Y$ be two nonempty convex subsets of real Hausdorff topological vector spaces, where $Y$ is compact, and let $F, G, H: X \rightrightarrows Y$ be three set-valued mappings with $F(x) \subset G(x) \subset H(x)$ for each $x \in X$ such that

(a) $F(x)$ and $H(x)$ are open in $Y$ for each $x \in X$, and $F^{-1}(y)$ and $H^{-1}(y)$ are convex for each $y \in Y$; and

(b) $G^{-1}(y)$ is open in $X$ for each $y \in Y$, and $Y \backslash G(x)$ is convex for each $x \in X$.

Then either there is $y_{0} \in Y$ such that $F^{-1}\left(y_{0}\right)=\emptyset$, or

$$
\bigcap_{y \in Y} H^{-1}(y) \neq \emptyset,
$$

where we denote $\Phi^{-1}(y):=\{x \in X: y \in \Phi(x)\}$ for a mapping $\Phi: X \rightrightarrows Y$.

Theorem A can be deduced by using the powerful method of barycentric subdivision. By employing the same direction of $\mathrm{Ha}$, we can derive the following refinement result.

(c) 2016 Lin and Lai. This article is distributed under the terms of the Creative Commons Attribution 4.0 International License (http://creativecommons.org/licenses/by/4.0/), which permits unrestricted use, distribution, and reproduction in any medium, provided you give appropriate credit to the original author(s) and the source, provide a link to the Creative Commons license, and indicate if changes were made. 
Theorem B Let $X, Y$ be two nonempty convex subsets of real Hausdorff topological vector spaces, where $Y$ is compact, and let $A, B, C, D, E, F, G: X \rightrightarrows Y$ be set-valued mappings with $A(x) \subset B(x) \subset C(x) \subset D(x) \subset E(x) \subset F(x) \subset G(x)$ for each $x \in X$ such that

(a) $A(x)$ and $E(x)$ are open in $Y$ for each $x \in X$, and $B^{-1}(y)$ and $F^{-1}(y)$ are convex for each $y \in Y$; and

(b) $D^{-1}(y)$ is open in $X$ for each $y \in Y$, and $Y \backslash C(x)$ is convex for each $x \in X$.

Then either there is $y_{0} \in Y$ such that $A^{-1}\left(y_{0}\right)=\emptyset$, or

$$
\bigcap_{y \in Y} G^{-1}(y) \neq \emptyset .
$$

As we mentioned above, it inspires and encourages us to apply the multihierarchical approach to minimax theorems for scalar set-valued mappings in Section 3 and for setvalued mappings in Section 4. In this paper, we will continue in this direction and create a generalized aspect.

\section{Fixed point theorems and whole intersection theorems}

The following theorem is a generalized form of Lemma 2.2 in [3].

Theorem 1 Let $X$ be a nonempty compact convex subset of a Hausdorff topological vector space, and $Y$ be an n-simplex. Suppose that set-valued mappings $P, Q, R, S, T: X \rightrightarrows Y$ with $P(x) \subset Q(x) \subset R(x) \subset S(x) \subset T(x)$ for each $x \in X$ satisfy the following conditions:

(i) $P^{-1}(y)$ is nonempty for each $y \in Y, Y \backslash Q(x)$ is convex for each $x \in X$, and $R^{-1}(y)$ is closed for each $y \in Y$; and

(ii) $S(x)$ is closed for each $x \in X$, and $T^{-1}(y)$ is convex for each $y \in Y$.

Iff $: X \rightarrow Y$ is continuous, then there are $x_{0} \in X$ and $y_{0} \in Y$ such that

$$
f\left(x_{0}\right)=y_{0}, \quad y_{0} \in T\left(x_{0}\right)
$$

Proof By the same directional process of Lemma 2.2 in [3], we can deduce the theorem. So, we leave the proof to the readers.

As a consequence of Theorem 1, we have the following fixed point theorem.

Corollary 1 Under the framework of Theorem 1 , in addition, if $Y=X$ is an n-simplex and $f$ is the identity mapping on $X$, then $T$ has a fixed point in $X$.

The following corollary is a variant form of Theorem 1 .

Corollary 2 Let $Y$ be a nonempty compact convex subset of a Hausdorff topological vector space, and $Z$ be an n-simplex. Suppose that set-valued mappings $P, Q, R, S, T: Z \rightrightarrows Y$ with $P(x) \subset Q(x) \subset R(x) \subset S(x) \subset T(x)$ for each $x \in Z$ satisfy the following conditions:

(i) $P(x)$ is nonempty for each $x \in Z, Z \backslash Q^{-1}(y)$ is convex for each $y \in Y$, and $R(x)$ is closed for each $x \in Z$; and

(ii) $S^{-1}(y)$ is closed for each $y \in Y$, and $T(x)$ is convex for each $x \in Z$.

If $: Y \rightarrow Z$ is continuous, then there are $x_{0} \in Z$ and $y_{0} \in Y$ such that

$$
f\left(y_{0}\right)=x_{0}, \quad y_{0} \in T\left(x_{0}\right)
$$


Now, we can prove Theorem B.

Proof of Theorem B Suppose that the first alternative is not true. Then $A^{-1}(y) \neq \emptyset$ for all $y \in Y$. For each $y \in Y$, there is $x_{y} \in A^{-1}(y)$ such that $y \in A\left(x_{y}\right)$. Therefore, $Y \subset \bigcup_{x \in X} A(x)$. By the compactness of $Y$ and since $A(x)$ is open for each $x \in X$, there exist $x_{1}, x_{2}, \ldots, x_{n} \in X$ such that

$$
Y \subset \bigcup_{i=1}^{n} A\left(x_{i}\right)
$$

Let $Z:=\operatorname{conv}\left\{x_{1}, x_{2}, \ldots, x_{n}\right\}$ where $\operatorname{conv} L$ means the convex hull of a set $L$, and let $\alpha_{1}, \alpha_{2}, \ldots, \alpha_{n}$ be a partition of unity on $Y$ subordinated to the cover $\left\{A\left(x_{i}\right): i=1,2, \ldots, n\right\}$. Define $f: Y \rightarrow X$ by $f(y):=\sum_{i=1}^{n} \alpha_{i}(y) x_{i}$. For each $y \in Y, 1 \leq i \leq n$, if $\alpha_{i}(y)>0, y \in A\left(x_{i}\right)$, then $x_{i} \in A^{-1}(y) \subset B^{-1}(y)$. By the convexity of $B^{-1}(y), f(y) \in B^{-1}(y)$, or

$$
y \in B(f(y))
$$

for all $y \in Y$.

On the other hand, define $P: Z \rightrightarrows Y$ by

$$
P(x):=Y \backslash G(x)
$$

for each $x \in Z$, and define $Q, R, S, T: Z \rightrightarrows Y$ similarly, with the mapping $G$ in (2) replaced by $F, E, D$, and $C$, respectively. Suppose that $\bigcap_{y \in Y} G^{-1}(y)=\emptyset$. Then $P(z) \neq \emptyset$ for each $z \in Z$. Therefore, by conditions (a) and (b), all conditions of Corollary 2 are valid for the mappings $P, Q, R, S$, and $T$. It follows that there are $x_{0} \in Z$ and $y_{0} \in Y$ such that $f\left(y_{0}\right)=x_{0}$ and $y_{0} \in T\left(x_{0}\right)$. Hence, $y_{0} \notin C\left(x_{0}\right)$, and so $y_{0} \notin B\left(x_{0}\right)=B\left(f\left(y_{0}\right)\right)$, which contracts (1). Therefore, the second alternative holds.

The following corollary is a variant form of Theorem B.

Corollary 3 Let $X, Y$ be nonempty convex subsets of real Hausdorff topological spaces, and $X$ be compact. Suppose that set-valued mappings $A, B, C, D, E, F, G: X \rightrightarrows Y$ possess the following conditions:

(i) $A^{-1}(y)$ is open in $X$ for each $y \in Y, B(x)$ is convex in $Y$ for each $x \in X$, and $X \backslash C^{-1}(y)$ is convex for each $y \in Y$;

(ii) $D(x)$ is open in $Y$ for each $x \in X, E^{-1}(y)$ is open in $X$ for each $y \in Y$, and $F(x)$ is convex in $Y$ for each $x \in X$; and

(iii) $A(x) \subset B(x) \subset C(x) \subset D(x) \subset E(x) \subset F(x) \subset G(x)$ for each $x \in X$.

Then either there is $x_{0} \in X$ such that $A\left(x_{0}\right)=\emptyset$, or

$$
\bigcap_{x \in X} G(x) \neq \emptyset
$$

The following two corollaries can be derived from Corollary 3.

Corollary 4 Under the framework of Corollary 3 , in addition, if $A=B, C=D$, and $E=F=$ $G$, then Corollary 3 is reduced to Theorem A. 
Corollary 5 Under the framework of Corollary 3 except the compactness of $X$, in addition, let $K \subset X$ be nonempty, compact, and convex. Then either there is $x_{0} \in K$ such that $A\left(x_{0}\right)=$ $\emptyset$, or

$$
\bigcap_{x \in X} G(x) \neq \emptyset
$$

\section{Multihierarchical structures for scalar set-valued mappings}

In this section, we present minimax theorems for scalar set-valued mappings under multihierarchical structures. We first recall some definitions. Let $V$ and $W$ be two nonempty sets equipped with some suitable topologies. Then we say that $\Phi: V \rightrightarrows W$ is upper semicontinuous on $V$ iff for each $v_{0} \in V$ and for every open set $\tilde{W}$ containing $\Phi\left(v_{0}\right)$, there exists a neighborhood $\tilde{V}$ of $v_{0}$ such that $\Phi(\tilde{V}) \subset \tilde{W}$. We say that $\Phi$ is lower semicontinuous on $V$ iff for each $v_{0} \in V$ and for every open set $\tilde{W}$ with $\Phi\left(v_{0}\right) \cap \tilde{W} \neq \emptyset$, there exists a neighborhood $\tilde{V}$ of $v_{0}$ such that $\Phi(v) \cap \tilde{W} \neq \emptyset$ for all $v \in \tilde{V}$. If $\Phi$ is both lower semicontinuous and upper semicontinuous on $V$, then we say that $\Phi$ is continuous on $V$. If $W$ is a Hausdorff topological vector space, $C$ is a closed convex pointed cone with int $C \neq \emptyset$ in $W$, and $V$ is a convex subset of a vector space, then we say that $\Phi: V \rightrightarrows W$ is convex [13] (concave [13], respectively) on $V$ iff for any $v_{1}, v_{2} \in V$ and any $\lambda \in[0,1]$, $\Phi\left(\lambda v_{1}+(1-\lambda) v_{2}\right) \subset \lambda \Phi\left(v_{1}\right)+(1-\lambda) \Phi\left(v_{2}\right)-C\left(\lambda \Phi\left(v_{1}\right)+(1-\lambda) \Phi\left(v_{2}\right) \subset \Phi\left(\lambda v_{1}+(1-\lambda) v_{2}\right)-C\right.$, respectively). We say that $\Phi: V \rightrightarrows Z$ is quasi-convex (quasi-concave, respectively) [13] on $V$ iff the set $\{v \in V: \Phi(v) \subset z-C\}(\{v \in V: \Phi(v) \subset z+C\}$, respectively) is convex for all $z \in Z$. Whenever $W=\mathbb{R}, C=\mathbb{R}_{+}$, and $\Phi$ becomes a single-valued function, then the above concepts of mappings coincide with the classical ones. We propose a proposition and several examples to illustrate some relations between those mappings.

Proposition 1 For a set-valued mapping, the convexity implies the quasi-convexity (Proposition 3.9 in [13]). However, the concavity does not imply the quasi-concavity.

Proof We only need propose a counterexample to explain the second statement. Let $X=$ $[0,1], C=\mathbb{R}_{+}$, and $\Phi: X \rightrightarrows \mathbb{R}$ be defined by

$$
\Phi(x):= \begin{cases}\{0\}, & x=0, \\ \left\{t:-2\left(2-x^{2}\right) \leq t \leq 2\left(2-x^{2}\right)\right\}, & x \neq 0 .\end{cases}
$$

The set

$$
L_{\Phi, \geq}(\theta):=\left\{x \in[0,1]: \Phi(x) \subset \theta+\mathbb{R}_{+}\right\}= \begin{cases}{[0,1],} & \theta \leq-4, \\ \{0\} \cup[\theta / 2-1,1], & -4<\theta \leq-2, \\ \{0\}, & -2<\theta \leq 0, \\ \emptyset, & \theta>0,\end{cases}
$$

is not convex for all $\theta \in \mathbb{R}$. Then $\Phi$ is not quasi-concave. On the other hand, since $c_{1} x_{2}^{2}+c_{2} x_{2}^{2} \geq\left(c_{1} x_{1}+c_{2} x_{2}\right)^{2}$ for all $c_{1}, c_{2} \in[0,1]$ and $x_{1}, x_{2} \in X, c_{1} \Phi\left(x_{1}\right)+c_{2} \Phi\left(x_{2}\right) \subset \Phi\left(c_{1} x_{1}+\right.$ $\left.c_{2} x_{2}\right)-\mathbb{R}_{+}$. Hence, $\Phi$ is concave.

Example 1 We would like to construct three set-valued mappings from $X \times Y$ to $\mathbb{R}$ that satisfy the following conditions: The first mapping is lower semicontinuous in the first 
variable but neither upper semicontinuous in the second variable nor quasi-convex in the first variable. The second mapping is quasi-convex in the first variable but not upper semicontinuous in the second variable. The third mapping is upper semicontinuous in the second variable but not lower semicontinuous in the first variable.

Sol. Let $X=Y=[0,1]$. We define

$$
\begin{aligned}
P(x, y) & := \begin{cases}{\left[-1+x^{2}, 1-x^{2}\right],} & x \neq 0, y \neq 0, \\
\left\{1-x^{2}\right\}, & x \neq 0, y=0, \\
\{0\} & \text { otherwise, }\end{cases} \\
Q(x, y): & = \begin{cases}{\left[-2+y^{2}, 2-y^{2}\right],} & y \neq 0, \\
\{0\}, & y=0,\end{cases} \\
R(x, y): & = \begin{cases}{\left[2-2\left(1-x^{2}\right), 2+2\left(1-x^{2}\right)\right],} & x \neq 0, y=0, \\
\{t: t \in[-2,6]\}, & x=0, y=0, \\
\{2\} & \text { otherwise. }\end{cases}
\end{aligned}
$$

Then we can confirm that $P(x, y)$ is a lower semicontinuous mapping in the first variable but neither upper semicontinuous in the second variable nor quasi-convex in the first variable; $Q(x, y)$ is quasi-convex in first variable but not upper semicontinuous in the second variable; and $R(x, y)$ is upper semicontinuous in the second variable but not lower semicontinuous in the first variable. Since the set

$$
L_{P, \leq}^{y}(\theta):=\left\{x \in[0,1]: P(x, y) \subset \theta-\mathbb{R}_{+}\right\}= \begin{cases}{[0,1],} & \theta \geq 1, \\ \{0\} \cup[\sqrt{1-\theta}, 1], & 0<\theta<1, \\ \{0,1\}, & \theta=0, \\ \emptyset, & \theta<0,\end{cases}
$$

is not convex for all $\theta \in \mathbb{R}$, the mapping $P(x, y)$ is not quasi-convex in the first variable. Nevertheless, since the set

$$
\begin{aligned}
L_{Q, \leq}^{y}(\theta): & =\left\{x \in[0,1]: Q(x, y) \subset \theta-\mathbb{R}_{+}\right\} \\
& = \begin{cases}{[0,1],} & y \in[0,1], \theta \geq 2-y^{2}, \text { or } 0 \leq \theta<2, y=0, \\
\emptyset & \text { otherwise, }\end{cases}
\end{aligned}
$$

is convex for all $\theta \in \mathbb{R}$, the mapping $Q(x, y)$ is quasi-convex in the first variable. We omit checking the other properties of the mappings $P, Q, R$ and leave them to the readers.

Example 2 We would like to construct two set-valued mappings from $X \times Y$ to $\mathbb{R}$ that satisfy following conditions: One is a mapping that is upper semicontinuous in the first variable but not concave in the second variable. The other mapping is concave in the second variable but not upper semicontinuous in the first variable.

Sol. Let $X=Y=[0,1]$. We define

$$
S(x, y):= \begin{cases}{\left[7-y^{2}, 9+y^{2}\right],} & x=0, \\ \{8\}, & x \neq 0,\end{cases}
$$




$$
T(x, y):= \begin{cases}\left\{t: 8-2\left(2-y^{2}\right) \leq t \leq 8+2\left(2-y^{2}\right)\right\}, & x=0, \\ \left\{t: 0 \leq t \leq\left(2-x^{2}\right)\left(8+2\left(2-y^{2}\right)\right)\right\}, & x \neq 0 .\end{cases}
$$

It is not difficult to check that $S$ is upper semicontinuous in the first variable but $T$ is not, so we only need to check that $T$ is concave in the second variable but $S$ is not. Indeed, for any $c_{1}, c_{2} \in[0,1]$ and $y_{1}, y_{2} \in[0,1]$, since $2 c_{1}\left(2-y_{1}^{2}\right)+2 c_{2}\left(2-y_{2}^{2}\right) \leq 2\left(2-\left(c_{1} y_{1}+c_{2} y_{2}\right)^{2}\right)$, we have $c_{1} T\left(x, y_{1}\right)+c_{2} T\left(x, y_{2}\right) \subset T\left(x, c_{1} y_{1}+c_{2} y_{2}\right)-\mathbb{R}_{+}$. This proves that $T$ is concave in the second variable. On the other hand, we observe that $c_{1} y_{1}^{2}+c_{2} y_{2}^{2} \not \leq\left(c_{1} y_{1}+c_{2} y_{2}\right)^{2}$, and thus $c_{1} S\left(0, y_{1}\right)+c_{2} S\left(0, y_{2}\right) \not \subset S\left(0, c_{1} y_{1}+c_{2} y_{2}\right)-\mathbb{R}_{+}$. So, $S$ is not concave in the second variable.

Example 3 We would like to construct two set-valued mappings from $X \times Y$ to $\mathbb{R}$ that satisfy following conditions: One is a mapping that is quasi-convex in the first variable but is not continuous in second variable. The other mapping is continuous in the second variable, and its union in the first variable is compact but not quasi-convex in the first variable.

Sol. Let $X=Y=[0,1]$, and let $U(x, y):=R(x, y)+22$ for all $(x, y) \in X \times Y$, which means that we lift $R$ up to 22 , and let $V(x, y):=\left[28,28+4\left(x-x^{2}\right) y^{2}\right]$, where $R$ is the same as in Example 1. Hence, $U$ is quasi-convex in the first variable, upper semicontinuous but not continuous in the second variable, and $V$ is continuous in the second variable, and its union $\bigcup_{x \in X} V(x, y)=\left[28,28+y^{2}\right]$ is compact but not quasi-convex in the first variable.

The following two lemmas will help us to derive the main results. These two lemmas describe some relationships of convexities between a scalar set-valued mapping and realvalued function.

Lemma 1 Suppose that $X$ is a nonempty convex subset of a topological vector space and $G: X \rightrightarrows \mathbb{R}$ is a set-valued mapping such that $\max G(x)$ exists for each $x \in X$. Then the mapping $G: X \rightrightarrows \mathbb{R}$ is quasi-convex if and only if the mapping $x \mapsto \max G(x)$ is a quasiconvex function.

Proof Suppose that $G: X \rightrightarrows \mathbb{R}$ is quasi-convex. We need to show that the set $\{x \in X$ : $\max G(x) \leq c\}$ is convex for each $c \in \mathbb{R}$. Indeed, for any $x_{1}, x_{2} \in\{x \in X: \max G(x) \leq c\}$ and $\lambda \in[0,1]$, we have

$$
G\left(x_{1}\right) \subset \max G\left(x_{1}\right)-\mathbb{R}_{+} \subset c-\mathbb{R}_{+}
$$

and

$$
G\left(x_{2}\right) \subset \max G\left(x_{2}\right)-\mathbb{R}_{+} \subset c-\mathbb{R}_{+} .
$$

By the quasi-convexity of $G$, we have that $\lambda x_{1}+(1-\lambda) x_{2}$ also belongs to the set $\{x \in X$ : $G(x) \subset c-\mathbb{R}\}$, that is, $G\left(\lambda x_{1}+(1-\lambda) x_{2}\right) \subset c-\mathbb{R}$. We note that $\max G\left(\lambda x_{1}+(1-\lambda) x_{2}\right) \in$ $G\left(\lambda x_{1}+(1-\lambda) x_{2}\right)$, and then $\lambda x_{1}+(1-\lambda) x_{2}$ also belongs to the set $\{x \in X: \max G(x) \leq c\}$, and thus, the last set is convex. Therefore, the mapping $x \mapsto \max G(x)$ is quasi-convex.

Conversely, if the mapping $x \mapsto \max G(x)$ is quasi-convex, we need to show that the set $\left\{x \in X: G(x) \subset c-\mathbb{R}_{+}\right\}$is convex for each $c \in \mathbb{R}$. Indeed, for any $x_{1}, x_{2} \in\{x \in X: G(x) \subset$ 
$\left.c-\mathbb{R}_{+}\right\}$and $\lambda \in[0,1]$, we have $G\left(x_{1}\right) \subset c-\mathbb{R}_{+}$and $G\left(x_{1}\right) \subset c-\mathbb{R}_{+}$. Thus, max $G\left(x_{1}\right) \leq c$ and $\max G\left(x_{1}\right) \leq c$. Since the mapping $x \mapsto \max G(x)$ is quasi-convex, $\max G\left(\lambda x_{1}+(1-\lambda) x_{2}\right) \leq$ $c$, and $G\left(\lambda x_{1}+(1-\lambda) x_{2}\right) \subset \max G\left(\lambda x_{1}+(1-\lambda) x_{2}\right)-\mathbb{R}_{+}$. Hence, $\lambda x_{1}+(1-\lambda) x_{2} \in\{x \in X$ : $\left.G(x) \subset c-\mathbb{R}_{+}\right\}$. Therefore, $G: X \mapsto \mathbb{R}$ is quasi-convex.

Lemma 2 Suppose that $X$ is a nonempty convex subset of a topological vector space and $G: X \rightrightarrows \mathbb{R}$ is a set-valued mapping such that $\max G(x)$ exists for each $x \in X$. Then the mapping $G: X \rightrightarrows \mathbb{R}$ is convex (concave, resp.) if and only if the mapping $x \mapsto \max G(x)$ is a convex (concave, resp.) function.

Proof We can deduce the conclusion directly from the definition.

With the help of Lemmas 1 and 2, we derive the following scalar hierarchical minimax theorem.

Theorem 2 Let $X, Y$ be two nonempty compact convex subsets of real Hausdorff topological vector spaces. Let $P, Q, R, S, T, U, V: X \times Y \rightrightarrows \mathbb{R}$ be set-valued mappings with nonempty compact values such that

$$
\begin{aligned}
\max P(x, y) & \leq \max Q(x, y) \leq \max R(x, y) \leq \max S(x, y) \\
& \leq \max T(x, y) \leq \max U(x, y) \leq \max V(x, y)
\end{aligned}
$$

for all $(x, y) \in X \times Y$ and that the set $\bigcup_{x \in X} V(x, y)$ is compact for all $y \in Y$. Suppose that the following conditions are satisfied:

(i) $y \mapsto V(x, y)$ is continuous on $Y$ for each $x \in X, x \mapsto U(x, y)$ is quasi-convex on $X$ for each $y \in Y, y \mapsto T(x, y)$ is concave for each $x \in X$, and $x \mapsto S(x, y)$ is upper semicontinuous on $X$ for each $y \in Y$;

(ii) $y \mapsto R(x, y)$ is upper semicontinuous on $Y$ for each $x \in X$, and $x \mapsto Q(x, y)$ is quasi-convex on $X$ for each $y \in Y$;

(iii) $x \mapsto P(x, y)$ is lower semicontinuous on $X$ for each $y \in Y$, and $y \mapsto P(x, y)$ is upper semicontinuous on $Y$ for each $x \in X$; and

(iv) for each $w \in Y$, there is $x_{w} \in X$ such that

$$
\max V\left(x_{w}, w\right) \leq \max \bigcup_{y \in Y} \min \bigcup_{x \in X} V(x, y)
$$

Then we have the following relation:

$$
\min \bigcup_{x \in X} \max \bigcup_{y \in Y} P(x, y) \leq \max \bigcup_{y \in Y} \min \bigcup_{x \in X} V(x, y) .
$$

Proof Suppose that $\max \bigcup_{y \in Y} \min \bigcup_{x \in X} V(x, y) \neq \infty$. For any $\alpha \in \mathbb{R}$ such that $\alpha>$ $\max \bigcup_{y \in Y} \min \bigcup_{x \in X} V(x, y)$, define $A: X \rightrightarrows Y$ by

$$
A(x)=\{y \in Y: \max V(x, y)<\alpha\}
$$

for all $x \in X$, and similarly $B, C, D, E, F$, and $G$, with the mapping $V$ inside the braces in (4) replaced by $U, T, S, R, Q$, and $P$, respectively. Since $\max U(x, y) \leq \max V(x, y)$ for all 
$(x, y) \in X \times Y, A(x) \subset B(x)$. Similarly, $B(x) \subset C(x) \subset D(x) \subset E(x) \subset F(x) \subset G(x)$ for each $x \in X$.

From the upper semicontinuity of the mappings $R, S, V$ and Lemma 2.5 in [13], the sets $A(x), D^{-1}(y)$, and $E(x)$ are open for all $x \in X$ and $y \in Y$. By the convexity of the mappings $Q, T$, and $U$ and Lemmas 1 and 2 , the sets $B^{-1}(y), F^{-1}(y)$, and $Y \backslash C(x)$ are convex for each $x \in X$ and for each $y \in Y$.

By condition (iv) the sets $A^{-1}(y) \neq \emptyset$ for each $y \in Y$. Hence, from Theorem B,

$$
\bigcap_{y \in Y} G^{-1}(y) \neq \emptyset
$$

Therefore, there exists $\xi \in X$ such that $\max P(\xi, y)<\alpha$ for all $y \in Y$. By Lemma 7 in [14] we know that $\max \bigcup_{y \in Y} P(\xi, y) \leq \alpha$. This implies that

$$
\min \bigcup_{x \in X} \max \bigcup_{y \in Y} P(x, y) \leq \alpha
$$

and hence relation (3) is valid. We complete the proof.

If we let all mappings be equal and single-valued in Theorem 2, then Theorem 2 can be compared to [4-7]. The following example shows that Theorem 2 is valid.

Example 4 Let us choose $P, Q, R, S, T, U, V$ the same as in Examples 1-3. Note that the inequality $\max P(x, y) \leq \max Q(x, y) \leq \max R(x, y) \leq \max S(x, y) \leq \max T(x, y) \leq$ $\max U(x, y) \leq \max V(x, y)$ is valid for all $(x, y) \in X \times Y$. Then, all conditions of Theorem 2 except condition (iv) are true. Let us check whether condition (iv) is true or not. Note that RHS of (iv) $=\max \bigcup_{y \in[0,1]} \min \bigcup_{x \in[0,1]}\left[28,28+4\left(x-x^{2}\right) y^{2}\right]=28$, and LHS of (iv) = $28+4\left(x_{y}-x_{y}^{2}\right) y^{2}$. In order to let the inequality LHS $\leq$ RHS hold, we can choose

$$
x_{y}= \begin{cases}\text { any point in }[0,1], & y=0, \\ 1 \text { or } 0, & y \neq 0 .\end{cases}
$$

Hence, condition (iv) of Theorem 2 comes true. Thus, all conditions of Theorem 2 are valid. Also, note that $\min \bigcup_{x \in X} \max \bigcup_{y \in Y} P(x, y)=\min \left\{0,1-x^{2}: x \in[0,1]\right\}=0$. Therefore, the conclusion of Theorem 2 holds.

In Example 4, we cannot apply Theorem 2 in [15] to confirm whether relation (3) is true or not. The reason is that $P \neq Q \neq R, S \neq T$, and $U \neq V$. If $P=Q=R=S=T=U=V$, then Theorem 2 can be reduced to Corollary 2 in [15].

\section{Multihierarchical structures for set-valued mappings}

In this section, we show that multihierarchical structures can work on Hausdorff topological vector spaces. Let $W$ be a Hausdorff topological vector space, and $C \subset W$ a closed convex and pointed cone with apex at the origin and int $C \neq \emptyset$. Let $C^{*}:=\left\{g \in W^{*}: g(c) \geq\right.$ 0 for all $c \in C$, where $W^{*}$ is the set of all continuous linear functionals on $W$. We also use the notations $\operatorname{Max} \Omega, \operatorname{Min} \Omega, \operatorname{Max}_{w} \Omega$, and $\operatorname{Min}_{w} \Omega$ for a nonempty set $\Omega$ in $W$. These notations represent the sets of maximal points, minimal points, weakly maximal points, 
and weakly minimal points of $\Omega$ [13], respectively. We also use the notation ' $F \preceq G$ ' to represent the relation

$$
\operatorname{Max}_{w} F \subset \operatorname{Max}_{w} G-C
$$

for two nonempty sets $F$ and $G$ in $W$. Applying the techniques of Theorem 3 in [15], we discuss the following minimax theorem with multihierarchical structures.

Theorem 3 Let $X, Y$ be nonempty compact convex subsets of real Hausdorff topological spaces, and $W$ be a complete locally convex Hausdorff topological vector space. Suppose that set-valued mappings $P, Q, R, S, T, U, V: X \times Y \rightrightarrows W$ with nonempty compact values satisfy the following conditions:

(i) $(x, y) \mapsto P(x, y)$ is upper semicontinuous on $X \times Y, y \mapsto R(x, y)$ is upper semicontinuous on $Y$ for each $x \in X, x \mapsto Q(x, y)$ is naturally quasi-convex [13], and $x \mapsto P(x, y)$ is lower semicontinuous on $X$ for each $y \in Y$;

(ii) $x \mapsto S(x, y)$ is upper semicontinuous on $X$ for each $y \in Y$, and $y \mapsto T(x, y)$ is concave on $Y$ for each $x \in X$;

(iii) $(x, y) \mapsto V(x, y)$ is upper semicontinuous on $X \times Y, x \mapsto U(x, y)$ is naturally quasi-convex on $X$ for each $y \in Y$, and $y \mapsto V(x, y)$ is continuous on $Y$ for each $x \in X$;

(iv) for any $\varphi \in C^{*}$ and $y \in Y$, there is $x_{y} \in X$ such that

$$
\max \varphi V\left(x_{y}, y\right) \leq \max \bigcup_{y \in Y} \min \bigcup_{x \in X} \varphi V(x, y)
$$

(v) for each $y \in Y$,

$$
\operatorname{Max} \bigcup_{y \in Y} \operatorname{Min}_{w} \bigcup_{x \in X} V(x, y) \subset \operatorname{Min}_{w} \bigcup_{x \in X} V(x, y)+C ;
$$

and

(vi) for all $(x, y) \in X \times Y, P(x, y) \preceq Q(x, y), Q(x, y) \preceq R(x, y), R(x, y) \preceq S(x, y)$, $S(x, y) \preceq T(x, y), T(x, y) \preceq U(x, y)$, and $U(x, y) \preceq V(x, y)$.

Then we have the relation

$$
\operatorname{Max} \bigcup_{y \in Y} \operatorname{Min}_{w} \bigcup_{x \in X} V(x, y) \subset \operatorname{Min}\left(\operatorname{conv} \bigcup_{x \in X} \operatorname{Max}_{w} \bigcup_{y \in Y} P(x, y)\right)+C .
$$

Proof Some techniques of the proof are similar to those of Theorem 3 in [15]. For sake of completeness, we will shorten its proof as follows.

Let $v \notin \operatorname{co}\left(\bigcup_{x \in X} \operatorname{Max}_{w} \bigcup_{y \in Y} P(x, y)\right)+C$. Then there is a nonzero continuous linear functional $g: W \rightarrow \mathbb{R}$ such that

$$
g(v)<\min \bigcup_{x \in X} \max \bigcup_{y \in Y} g P(x, y) .
$$

With the help of Propositions 3.9 and 3.13 in [13], conditions (i)-(iii) of Theorem 2 are satisfied for the mappings $g P, g Q, g R, g S, g T, g U, g V$. By Proposition 2(a) in [15] and con- 
dition (vi),

$$
g P(x, y) \leq g Q(x, y) \leq g R(x, y) \leq g S(x, y) \leq g T(x, y) \leq g U(x, y) \leq g V(x, y)
$$

for all $(x, y) \in X \times Y$. Combining this and condition (iv) gives that all conditions of Theorem 2 hold for the mappings $g P, g Q, g R, g S, g T, g U, g V$. Hence, by Theorem 2,

$$
g(v)<\max \bigcup_{y \in Y} \min \bigcup_{x \in X} g V(x, y)
$$

Since $Y$ is compact, there is $y^{\prime} \in Y$ such that

$$
v \notin \operatorname{Min}_{w} \bigcup_{x \in X} V\left(x, y^{\prime}\right)+C
$$

By (v),

$$
v \notin \operatorname{Max} \bigcup_{y \in Y} \operatorname{Min}_{w} \bigcup_{x \in X} V(x, y) .
$$

This means that, for every $v \in \operatorname{Max} \bigcup_{y \in Y} \operatorname{Min}_{w} \bigcup_{x \in X} V(x, y)$,

$$
v \in \operatorname{co}\left(\bigcup_{x \in X} \operatorname{Max}_{w} \bigcup_{y \in Y} P(x, y)\right)+C
$$

Therefore, $(\mathrm{H})$ is valid.

The following example is very suitable to illustrate Theorem 3.

Example 5 Let $X=Y=[0,1], W=\mathbb{R}^{2}$, and $C=\mathbb{R}_{+}^{2}$. Define the set-valued mappings $P, Q, R, S, T, U, V: X \times Y \rightrightarrows W$ by

$$
\begin{aligned}
& P(x, y):= \begin{cases}\{(x, 1)\}, & y \neq 0, \\
\{(x, t): t \in[-1,1]\}, & y=0,\end{cases} \\
& Q(x, y):= \begin{cases}\{(s, 1): s \in[-1,1]\}, & x=0, y=0, \\
\left\{(s, t): s \in[-1,1], t \in\left[-2+y^{2}, 2-y^{2}\right]\right\}, & x=0, y \neq 0, \\
\{(1,1)\}, & x \neq 0, y=0, \\
\left\{(1, t): t \in\left[-2+y^{2}, 2-y^{2}\right]\right\}, & x \neq 0, y \neq 0,\end{cases} \\
& R(x, y):= \begin{cases}\{(1, t): t \in[-2,2]\}, & x=0, y=0, \\
\{(1,2)\}, & x=0, y \neq 0, \\
\left\{\left(2-x^{2}, t\right): t \in[-2,2]\right\}, & x \neq 0, y=0, \\
\left\{\left(2-x^{2}, 2\right)\right\}, & x \neq 0, y \neq 0,\end{cases} \\
& S(x, y):= \begin{cases}\left\{(s, t): s \in[0,2], t \in\left[1,2+y^{2}\right]\right\}, & x=0, \\
\left\{(2, t): t \in\left[1,2+y^{2}\right]\right\}, & x \neq 0,\end{cases} \\
& T(x, y):= \begin{cases}\left\{\left(s, 4-y^{2}\right): s \in[-2,2]\right\}, & x=0, \\
\left\{\left(s, 4-y^{2}\right): s \in\left[0,3-x^{2}\right]\right\}, & x \neq 0,\end{cases}
\end{aligned}
$$




$$
U(x, y):= \begin{cases}\{(5,4)\}, & x=0, y=0, \\ \{(5, t): t \in[0,4]\}, & x=0, y \neq 0, \\ \left\{(s, 4): s \in\left[3,4+(x-1 / 2)^{2}\right]\right\}, & x \neq 0, y=0, \\ \left\{(s, t): s \in\left[3,4+(x-1 / 2)^{2}\right], t \in[0,4]\right\}, & x \neq 0, y \neq 0,\end{cases}
$$

and

$$
V(x, y):=\left\{\left(5+x^{2}, t\right): t \in\left[y^{2}+4,5\right]\right\}
$$

for all $(x, y) \in X \times Y$.

We can check carefully by the definitions that the continuity and convexity hold for all the mappings $P, Q, R, S, T, U, V$. Therefore, conditions (i)-(iii) of Theorem 3 are valid. For any $y \in Y$ and $\left(g_{1}, g_{2}\right) \in C^{*}$,

$$
\max g V(x, y)=\max \left\{\left(5+x^{2}\right) g_{1}+\operatorname{tg}_{2}: y^{2}+4 \leq t \leq 5\right\}=\left(5+x^{2}\right) g_{1}+5 g_{2} .
$$

On the other hand,

$$
\max \bigcup_{y \in Y} \min \bigcup_{x \in X} g V(x, y)=\max \bigcup_{y \in Y}\left\{5 g_{1}+\left(y^{2}+4\right) g_{2}\right\}=5 g_{1}+5 g_{2} .
$$

For each $y \in Y$, if we choose

$$
x_{y}= \begin{cases}\text { any point in }[0,1], & g_{1}=0, \\ 0, & g_{1} \neq 0,\end{cases}
$$

then condition (iv) of Theorem 3 is valid. Since

$$
\begin{aligned}
\operatorname{Max} \bigcup_{y \in Y} \operatorname{Min}_{w} \bigcup_{x \in X} V(x, y) & =\{(5,6)\} \\
& \subset\left(\{5\} \times\left[y^{2}+4,5\right]\right) \cup\left([5,6] \times\left\{y^{2}+4\right\}\right)+C \\
& =\operatorname{Min}_{w} \bigcup_{x \in X} V(x, y)+C,
\end{aligned}
$$

condition (v) of Theorem 3 is true. Condition (vi) of Theorem 3 is valid by the following observations:

$$
\begin{aligned}
& \operatorname{Max}_{w} P(x, y)= \begin{cases}\{(x, 1)\}, & y \neq 0, \\
\{x\} \times[-1,1], & y=0,\end{cases} \\
& \operatorname{Max}_{w} Q(x, y)= \begin{cases}{[-1,1] \times\{1\},} & x=0, y=0, \\
\{(1,1)\}, & x \neq 0, y=0, \\
{[-1,1] \times\left[-2+y^{2}, 2-y^{2}\right],} & x=0, y \neq 0, \\
\{1\} \times\left[-2+y^{2}, 2-y^{2}\right], & x \neq 0, y \neq 0,\end{cases} \\
& \operatorname{Max}_{w} R(x, y)= \begin{cases}\{1\} \times[-2,2], & x=0, y=0, \\
\left\{2-x^{2}\right\} \times[-2,2], & x \neq 0, y=0, \\
\{(1,2)\}, & x=0, y \neq 0, \\
\left\{\left(2-x^{2}, 2\right)\right\}, & x \neq 0, y \neq 0,\end{cases}
\end{aligned}
$$




$$
\begin{aligned}
& \operatorname{Max}_{w} S(x, y)= \begin{cases}\{2\} \times\left[1,2+y^{2}\right], & x \neq 0, \\
\left([0,2] \times\left\{2+y^{2}\right\}\right) \cup\left(\{2\} \times\left[1,2+y^{2}\right]\right), & x=0,\end{cases} \\
& \operatorname{Max}_{w} T(x, y)= \begin{cases}\left\{\left(s, 4-y^{2}\right): s \in[-2,2]\right\}, & x=0, \\
\left\{\left(s, 4-y^{2}\right): s \in\left[0,3-x^{2}\right]\right\}, & x \neq 0,\end{cases}
\end{aligned}
$$

and

$$
\operatorname{Max}_{w} V(x, y)= \begin{cases}\{(5,4)\}, & x=0, y=0 \\ {\left[3,4+(x-1 / 2)^{2}\right] \times\{4\},} & x \neq 0, y=0 \\ \{5\} \times[0,4], & x=0, y \neq 0 \\ \left(\left[3,4+(x-1 / 2)^{2}\right] \times\{4\}\right) \cup\left(\left\{4+(x-1 / 2)^{2}\right\} \times[0,4]\right), & x \neq 0, y \neq 0\end{cases}
$$

Thus, all conditions of Theorem 3 are satisfied for the seven mappings $P, Q, R, S, T, U$, and $V$. By Theorem 3, relation (H) must be true. Indeed,

$$
\begin{aligned}
\operatorname{Max} \bigcup_{y \in Y} \operatorname{Min}_{w} \bigcup_{x \in X} V(x, y) & =\{(5,6)\} \\
& \subset\{(0,-1)\}+C \\
& =\operatorname{Min}\left(\operatorname{conv} \bigcup_{x \in X} \operatorname{Max}_{w} \bigcup_{y \in Y} P(x, y)\right)+C .
\end{aligned}
$$

\section{Conclusions}

We construct successfully minimax theorems with multihierarchical procedures of setvalued mappings. Just like the rainbow has seven colors, the multihierarchical methodology can work by seven set-valued mappings. The multihierarchical structure can include some particular cases that appeared in the literatures.

Competing interests

The authors declare that they have no competing interests.

\section{Authors' contributions}

The first author made substantial contributions to conception, design, and analysis and derived the drafting theories. The second author has been involved in revising it critically for important intellectual content. Both authors have given final approval of the version to be published.

\section{Author details}

${ }^{1}$ Department of Occupational Safety and Health, College of Public Health, China Medical University, Taichung, 40402,

Taiwan. ${ }^{2}$ Department of Mathematics, National Tsing Hua University, Hsinchu, 30013, Taiwan.

\section{Acknowledgements}

The first author was partially supported by grant MOST104-2115-M-039-001 of the Ministry of Science and Technology of Taiwan (Republic of China), which is gratefully acknowledged.

Received: 31 December 2015 Accepted: 25 August 2016 Published online: 31 August 2016

\section{References}

1. Fan, K: Fixed-point and minimax theorems in locally convex topological linear spaces. Proc. Natl. Acad. Sci. USA 38, 121-126 (1952)

2. Fan, K: Minimax theorems. Proc. Natl. Acad. Sci. USA 39, 42-47 (1953)

3. Ha, CW: A minimax theorem. Acta Math. Hung. 101, 149-154 (2003)

4. Liu, FC: A note on the von Neumann-Sion minimax principle. Bull. Inst. Math. Acad. Sin. 6, 517-524 (1978)

5. Komiya, H: Elementary proof for Sion's minimax theorem. Kodai Math. J. 11, 5-7 (1988)

6. Sion, M: On general minimax theorems. Pac. J. Math. 8, 171-176 (1958)

7. Terkelsen, F: Some minimax theorems. Math. Scand. 31, 405-413 (1972)

8. Kindler, J: Two-function topological minimax theorems. J. Math. Anal. Appl. 225, 312-325 (1998) 
9. Chen, $\mathrm{CM}$, Chang, TH: Some results for the family KKM(X,Y) and the $\Phi$-mapping. J. Math. Anal. Appl. 329, 92-101 (2007)

10. Chang, SS, Cao, S, Wu, X, Wang, D: Some nonempty intersection theorems in generalized interval spaces with applications. J. Math. Anal. Appl. 199, 787-803 (1996)

11. Zhang, J: Some minimax inequalities for mappings with noncompact domain. Appl. Math. Lett. 17, 717-720 (2004)

12. Shioji, N: A further generalization of the Knaster-Kuratowski-Mazurkiewicz theorem. Proc. Am. Math. Soc. 111 187-195 (1991)

13. Lin, YC, Ansari, QH, Lai, HC: Minimax theorems for set-valued mappings under cone-convexities. Abstr. Appl. Anal. (2012). doi:10.1155/2012/310818

14. Lin, YC, Pang, C-T: The hierarchical minimax inequalities for set-valued mappings. Abstr. Appl. Anal. (2014) doi:10.1155/2014/190821

15. Lin, YC: Minimax problems under hierarchical structures. J. Inequal. Appl. (2015). doi:10.1186/s13660-015-0575-X

\section{Submit your manuscript to a SpringerOpen ${ }^{\circ}$} journal and benefit from:

- Convenient online submission

Rigorous peer review

- Immediate publication on acceptance

Open access: articles freely available online

- High visibility within the field

- Retaining the copyright to your article 